\title{
Smart Detection Using the Cascaded Artificial Neural Network for OFDM with Subcarrier Number Modulation
}

\author{
Jun Li, Member, IEEE, Shuping Dang, Member, IEEE, Miaowen Wen, Senior Member, IEEE, \\ Zhenrong Zhang, Member, IEEE, and Qiang Li
}

\begin{abstract}
Since the proposal of orthogonal frequency-division multiplexing with subcarrier number modulation (OFDM-SNM), the high detection complexity of this novel modulation scheme becomes a major issue degrading its applicability in practice. To provide an easy-to-implement and low-complexity solution to the detection of the OFDM-SNM signal, we design a cascaded artificial neural network (CANN) at the OFDM-SNM receiver to decouple the detection of subcarrier number pattern (SNP) and constellation symbols on the active subcarriers. This is the first time that the CANN design is introduced to assist in detecting parallel signals. Numerical simulations verify the effectiveness and efficiency of the proposed smart detection scheme and show that the CANN based smart detection with adequate training can yield comparable error performance to the optimal maximum-likelihood detection at much lower complexity.
\end{abstract}

Index Terms-Artificial neural network (ANN), orthogonal frequency-division multiplexing (OFDM), subcarrier number modulation, low-complexity detection, cascaded architecture.

\section{INTRODUCTION}

$\mathbf{O}$ RTHOGONAL frequency-division multiplexing (OFDM) has been implemented in many application scenarios to generate parallel transmissions for achieving high data rates with the proliferation of fourth generation $(4 \mathrm{G})$ communication networks [1]. To further explore the spectral and energy efficiency of OFDM and mitigate the high peak-to-average power ratio (PAPR) problem, various frequency-domain sparsity modulation schemes without activating all available frequency resources have been proposed and analyzed in recent years [2]-[5].

Among these schemes, OFDM with subcarrier number modulation (OFDM-SNM) introduces an extra signal modulation dimension called the number dimension in addition to the classic ampli-

This work was supported in part by National Natural Science Foundation of China under Grants 61872102 and 61661004 , in part by the International Collaborative Research Program of Guangdong Science and Technology Department under Grant 2020A0505100061, in part by the Pearl River Nova Program of Guangzhou under Grant 201806010171, in part by the Fundamental Research Funds for the Central Universities under Grant 2019SJ02, and in part by the Guangxi Science Key Research and Development Project under Grant AB1850043 (Corresponding author: Qiang Li).

Jun Li is Research Center of Intelligent Communication Engineering, School of Electronics and Communication Engineering, Guangzhou University, Guangzhou 510006, China (e-mail: lijun52018@gzhu.edu.cn).

Shuping Dang is with Computer, Electrical and Mathematical Science and Engineering Division, King Abdullah University of Science and Technology (KAUST), Thuwal 23955-6900, Saudi Arabia (e-mail: shuping.dang@kaust.edu.sa).

Miaowen Wen is with the School of Electronic and Information Engineering, South China University of Technology, Guangzhou 510641, China (e-mail: eemwwen@scut.edu.cn).

Zhenrong Zhang is with the School of Computer, Electronics and Information, Guangxi University, and Guangxi Key Laboratory of Multimedia Communications and Network Technology, Nanning 530004, China (e-mail: zzr76@gxu.edu.cn).

Qiang Li is with the College of Information Science and Technology, Jinan University, Guangzhou 510632, China (e-mail: qiangli@jnu.edu.cn). tude and phase dimensions and signifies additional information in the number of active subcarriers for each transmission attempt [6]. The performance advantage of OFDM-SNM over plain OFDM has been rigorously proven for certain application scenarios. Analytical and numerical results show that OFDM-SNM is particularly suited for simple OFDM systems using a low-order amplitude-phase modulation (APM) scheme [7], e.g., binary phase shift keying (BPSK) and quadrature phase shift keying (QPSK), and with limited transmit power [8]. These features make OFDM-SNM an ideal modulation candidate for the low-complexity wireless applications in the Internet of Things (IoT) [9].

On the other hand, OFDM-SNM introduces an additional modulation dimension and inevitably results in an increasing amount of detection complexity, albeit with advantages on spectral and energy efficiency under special circumstances. This computing disadvantage could degrade the applicability of OFDM-SNM in practice for delay-critical wireless applications and/or real-time data services. In theoretic studies, maximum-likelihood (ML) detection is employed to detect the subcarrier number pattern (SNP) together with the carried constellation symbols on the active subcarriers as a whole, which leads to the optimal detection performance, while resulting in exponentially high detection complexity [10]. To reduce the detection complexity, several low-complexity detection schemes have been proposed for OFDM-SNM and its alternatives [10]. However, numerical results have shown that they are still far from the optimal detection performance. More importantly, canonical low-complexity detection schemes, e.g., log-likelihood ratio (LLR) detection and energy detection, relying on per-subcarrier estimation criteria proposed for OFDM-IM cannot be directly applied to OFDM-SNM, because the number of active subcarriers by OFDM-SNM is varying. As a result, detection thresholds must be well designed by certain methods for all subcarriers depending on per-subcarrier channel state information (CSI). Such a design process is impractical for fast-changing transmission environments and will inevitably incur another dimension of optimization.

To mitigate the aforementioned issues of low-complexity detection designs and provide near-optimal detection for OFDMSNM with low computational complexity, we herein propose a smart detection scheme using a cascaded artificial neural network (CANN) design. The CANN is a novel neural network architecture connecting multiple ANNs in a cascaded manner, which is capable of realizing much more sophisticated processing than a plain ANN. In communication science, the CANN has been applied at the transmitter to harvest a spatial diversity gain [11], while this is the first work, to the best of the authors' knowledge, that utilizes the CANN at the receiver to assist on signal detection. As shown by the numerical results in this letter, the CANN based smart detection is able to achieve comparable performance to the ML 
detection, while reducing the computational complexity from the exponential scale to the polynomial scale.

\section{SYSTEM MODEL}

Considering a typical OFDM system with $N$ subcarriers $^{1}, k \in$ $\mathcal{N}=\{1,2, \ldots, N\}$ subcarriers are activated during each channel use to form an SNP signifying a bit stream with $p_{\mathrm{SNP}}=\log _{2}(N)$ bits. Furthermore, for a given number of active subcarriers $k$, there will also be $k$ constellation symbols conveyed on the $k$ subcarriers. Assuming $M$-ary PSK ( $M$-PSK) is used as the APM scheme, these constellation symbols can represent another bit stream with $p_{\mathrm{APM}}(k)=k \log _{2}(M)$ bits. Abiding the maximum entropy principle, we assume all incoming bits are independently distributed over $\{0,1\}$ with equal probabilities. Both SNP and constellation symbols can be organized by a successive padding procedure and mapped to an OFDM transmission block in a bijective way.

We now show in detail how both SNP and constellation symbols are jointly mapped to a unique OFDM transmission block by the successive padding procedure. The $k$ th SNP with $k$ active subcarriers can be uniquely represented by the vector $\mathbf{x}_{\mathrm{SNP}}(k)=\left[\phi_{1}, \phi_{2}, \ldots, \phi_{N}\right]^{T} \in\{0,1\}^{N \times 1}$, where $(\cdot)^{T}$ denotes the transpose operation of the matrix/vector enclosed; $\phi_{n} \in\{0,1\}$ denotes the on/off activation state of the $n$th subcarrier. Without loss of generality, we adopt a sequential on/off activation state assignment ${ }^{2}$ in this letter and therefore have $\phi_{1}=\phi_{2}=\cdots=$ $\phi_{k}=1$ and $\phi_{k+1}=\phi_{k+2}=\cdots=\phi_{N}=0$. With $k$ active subcarriers, there are $k$ constellation symbols conveyed on the corresponding subcarriers, which are represented by the vector $\mathbf{x}_{\mathrm{APM}}(k)=\left[s_{1}, s_{2}, \ldots, s_{k}\right]^{T} \in \mathcal{M}^{k \times 1}$, where $\mathcal{M}$ is the set of $M$-ary constellation symbols; $s_{\kappa}$ is a certain $M$-ary complex symbol, $\forall \kappa=1,2, \ldots, k$. We normalize $\mathbb{E}\left\{\left(s_{\kappa}\right)^{H} s_{\kappa}\right\}=1$, where $(\cdot)^{H}$ denotes the conjugate transpose operation of the matrix/vector enclosed. Hence, we can write $s_{\kappa}=\exp \left(j \theta_{\kappa}\right)$, where $\theta_{\kappa}=\frac{2 \pi}{M}(m-1), \forall m=1,2, \cdots, M$ and $\kappa=1,2, \ldots, k$ for $M$-PSK.

Having $\mathbf{x}_{\mathrm{SNP}}(k)$ and $\mathbf{x}_{\mathrm{APM}}(k)$, the OFDM transmission block can be built by performing an $N$-point IFFT and appending a sufficiently long cyclic prefix as well as the successive padding as $\mathbf{x}(k)=\left[x_{1}, x_{2}, \ldots, x_{N}\right]^{T} \in(\{0\} \bigcup \mathcal{M})^{N \times 1}$, where $x_{n}=s_{n}$ for $n \leq k$, or $x_{n}=\phi_{n}=0$ otherwise. Because of the normalization of each symbol conveyed on the active subcarrier, obviously, we have $\mathbb{E}\left\{(\mathbf{x}(k))^{H} \mathbf{x}(k)\right\}=k$.

By removing cyclic prefix and performing FFT at the receiver, the multi-carrier signals transmitted over a dispersive channel can be converted to independently faded signals transmitted over parallel channels, resulting in the received OFDM block

$$
\mathbf{y}(k)=\left[y_{1}, y_{2}, \ldots, y_{N}\right]^{T}=\sqrt{P_{T} / k} \mathbf{H x}(k)+\mathbf{w} \in \mathbb{C}^{N \times 1},
$$

where $P_{T}$ is the total transmit power uniformly allocated over $k$ active subcarriers; $\mathbf{H}=\operatorname{diag}\left\{h_{1}, h_{2}, \ldots, h_{N}\right\}$ is the diagonal

\footnotetext{
${ }^{1}$ As inverse fast Fourier transform (IFFT) is in use to generate the OFDM transmission block for modern multi-carrier communication systems, $N$ is in general a power of two, i.e., 2, 4, 8, and etc. We also follow this setting in this letter to simplify our discussion.

${ }^{2}$ More complicated and efficient activation state assignment based on CSI can be found in [7], in which $k$ active subcarriers with higher channel power gains are selected to be activated.
}

channel coefficient matrix ${ }^{3}$, and $\mathbf{w}=\left[w_{1}, w_{2}, \ldots, w_{N}\right]^{T}$ is the frequency-domain additive white Gaussian noise (AWGN) vector with average noise power $N_{0}$ for each of its entry. According to the successive padding procedure, we can rewrite (1) by following the entry-wise input-output relation as $y_{n}=\sqrt{P_{T} / k} h_{n} s_{n}+w_{n}$ for $n \leq k$, or $y_{n}=w_{n}$ otherwise.

\section{Smart Detector Design Using the CANN}

\section{A. Information-Theoretic Basics}

Because of the bijective mapping relation between $\mathbf{x}_{\mathrm{SNP}}(k)$, $\mathbf{x}_{\mathrm{APM}}(k)$, and $\mathbf{x}(k)$ resulted by the successive padding procedure, it is clear that one can obtain the full information of $\mathbf{x}(k)$ as long as the information of $\mathbf{x}_{\mathrm{SNP}}(k)$ and $\mathbf{x}_{\mathrm{APM}}(k)$ has been perfectly known. Following this reasoning, we can derive the informationtheoretic derivation of the mutual information between $\mathbf{x}(k)$ and $\mathbf{y}(k)$ infra:

$$
\begin{aligned}
& I(\mathbf{x}(k) ; \mathbf{y}(k))=I\left(\mathbf{x}_{\mathrm{SNP}}(k), \mathbf{x}_{\mathrm{APM}}(k) ; \mathbf{y}(k)\right) \\
& \stackrel{(\mathrm{a})}{=} I\left(\mathbf{x}_{\mathrm{APM}}(k) ; \mathbf{y}(k) \mid \mathbf{x}_{\mathrm{SNP}}(k)\right)+I\left(\mathbf{x}_{\mathrm{SNP}}(k) ; \mathbf{y}(k)\right),
\end{aligned}
$$

where (a) is derived by the chain rule for mutual information [12]. The elegant relation derived in (2) naturally leads to a hint that an OFDM-SNM detector might perform the signal detection by two steps. In the first step, the OFDM-SNM detector can simply evaluate $\mathbf{x}_{\mathrm{SNP}}(k)$ by the knowledge of $\mathbf{y}(k)$. Then, with the estimate of $\mathbf{x}_{\mathrm{SNP}}(k)$ as prior knowledge, the OFDM-SNM detector performs the second-stage estimation of $\mathrm{x}_{\mathrm{APM}}(k)$ by the knowledge of $\mathbf{y}(k)$. From the basics of information theory, it is easy to know that assuming the guaranteed detection of $\mathbf{x}_{\mathrm{SNP}}(k)$, the detection of $\mathbf{x}_{\mathrm{APM}}(k)$ by $\mathbf{y}(k)$ should not be worse than the detection of $\mathbf{x}(k)$ by $\mathbf{y}(k)$. The above information-theoretic interpretation is the foundation of our proposed smart detector design.

\section{B. Framework of the Smart Detector}

Adhering to the information-theoretic basics given in the last subsection, we design a two-stage smart detector using a CANN architecture that splits the integrated detection procedure of $\mathbf{x}(k)$ into two sequential detection procedures of $\mathbf{x}_{\mathrm{SNP}}(k)$ and $\mathbf{x}_{\mathrm{APM}}(k)$. The framework of the smart detector is shown in Fig. 1. By processing the received OFDM block throughout the smart detector assisted by one primary ANN and $N$ secondary ANNs as well as a set of functional modules without intelligent autonomy, the transmission block $\mathbf{x}(k)$ can be intelligently estimated to be $\tilde{\mathbf{x}}(k)=\left[\tilde{x}_{1}, \tilde{x}_{2}, \ldots, \tilde{x}_{N}\right]^{T}$.

\section{Conventional Module Specifications}

To facilitate the implementation of the proposed smart detector, we provide the specifications of the constituent functional modules without intelligent autonomy in this subsection.

1) Pre-processing unit: The pre-processing unit is the same functional module as used in canonical OFDM systems, which splits the received OFDM block into $N$ non-dispersive and orthogonal signals $\left\{y_{n}\right\}$ eliminating inter-symbol interference.

\footnotetext{
${ }^{3}$ In this letter, we assume that the instantaneous CSI represented by $\mathbf{H}$ is perfectly known at the receiver.
} 


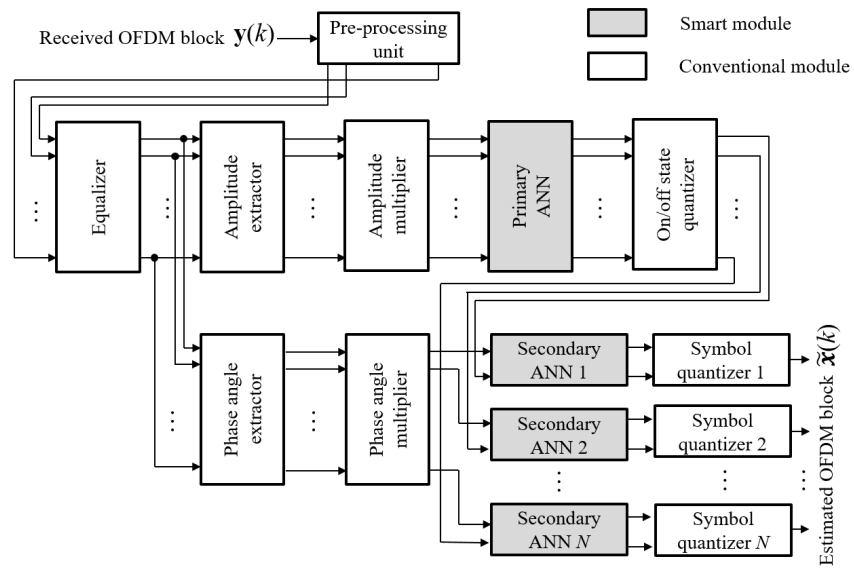

Fig. 1: Framework of the proposed smart detector using the CANN.

2) Equalizer: With the perfect knowledge of $\mathbf{H}$, the equalizer is employed to counteract the multiplicative distortion $\left\{h_{n}\right\}$ on each subcarrier rendered by channel fading. A zero-forcing equalizer can be implemented on each subcarrier to divide the orthogonal signal $y_{n}$ by $h_{n}$ and yields $\hat{x}_{n}=\hat{A}_{n} \exp \left(j \hat{\theta}_{n}\right)=y_{n} / h_{n}=$ $\left(\sqrt{P_{T} / k}\right) x_{n}+w_{n} / h_{n}$ and $\hat{x}_{n}=w_{n} / h_{n}$ for subcarriers with on and off states, respectively, where $\hat{A}_{n}$ and $\hat{\theta}_{n}$ are the amplitude and phase angle of the equalized symbol or null conveyed on the $n$th subcarrier with additive distortion.

3) Amplitude and phase angle extractors: These two extractors are used to obtain the amplitudes and phase angles of the equalized symbols or nulls, i.e., $\left\{\hat{A}_{n}\right\}$ and $\left\{\hat{\theta}_{n}\right\}$.

4) Amplitude and phase angle multipliers: These two multipliers are prior to the primary and secondary ANNs, aiming at restricting the ranges of inputs to the ANNs. In this way, the convergence of neural computing can be to some extent guaranteed. In particular, the amplitude multiplier multiples $\left\{\hat{A}_{n}\right\}$ by a constant factor $1 / \sqrt{P_{T}}$; the phase angle multiplier multiples $\left\{\hat{\theta}_{n}\right\}$ by a constant factor $1 /(2 \pi)$.

5) On/off state quantizer: Because the outputs of the primary ANN are generally decimals between 0 and 1, they can hardly be used as on/off state indicators for the next-stage computing without quantization. Denoting the $N$ outputs of the primary ANN as $\left\{\hat{\phi}_{n}\right\}$, the on/off state quantizer performs the manipulation on $\left\{\hat{\phi}_{n}\right\}$ and gives $\left\{\tilde{\phi}_{n}\right\}=\left\{\left\lfloor\hat{\phi}_{n}+1 / 2\right\rfloor\right\}$, where $\lfloor\cdot\rfloor$ denotes the floor function rounding the enclosed to the nearest integer less than or equal to the enclosed. We denote $\tilde{\mathbf{x}}_{\mathrm{SNP}}(k)=\left[\tilde{\phi}_{1}, \tilde{\phi}_{2}, \ldots, \tilde{\phi}_{N}\right]^{T}$ as the estimate of SNP.

6) Symbol quantizer: Because the output values of ANNs employing the logistic function (details are given in the next subsection) as the activation function are within $(0,1)$, the $n$th symbol quantizer first needs to project the outputs back to the open domain of the definition of sinusoidal functions, i.e., $(-1,1)$. Then, the estimated projected sine and cosine of the phase angle are used as imaginary and real parts for the symbol construction. Subsequently, the constructed symbol based on the estimates is mapped to an admissible symbol or the origin in the complex plane with the smallest Euclidean distance in a per-subcarrier manner, denoted by $\tilde{x}_{n}$. The output $\tilde{\mathbf{x}}(k)=\left[\tilde{x}_{1}, \tilde{x}_{2}, \ldots, \tilde{x}_{N}\right]^{T}$ from $N$ symbol quantizers is the final result of the smart detector that estimates the original transmission OFDM block $\mathbf{x}(k)$.

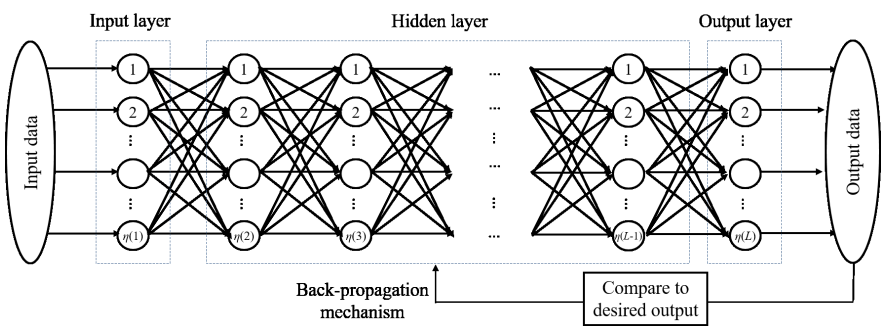

Fig. 2: A classic fully-connected ANN architecture using the backpropagation mechanism.

\section{ANN Functionality and Architecture Designs}

In this letter, we adopt a classic fully-connected architecture for both ANNs as shown in Fig. 2 and equip the ANNs with the neural computing functionality based on the back-propagation mechanism [13]. The back-propagation mechanism allows fine adjustments to neural computing parameters. For each ANN with a predetermined architecture design characterized by the number of layers $L$ and the number of neurons $\{\eta(l)\}$ in the lth layer, $\forall l \in\{1,2, \ldots, L\}$, there are two computing hyper-parameters required to be set beforehand, which are the learning rate $\alpha$ and the range expansion coefficient $\beta$ for randomly initializing link weights $\{\mathrm{W}(\nu(l), \nu(l+1))\}$ and activation thresholds $\{\mathrm{T}(\nu(l))\}$, where $\nu(l) \in\{1,2, \ldots, \eta(l)\}$ represents the index of an arbitrary neuron in the $l$ th layer. In particular, the random initialization process can be carried out by randomly assigning different uniformly distributed real numbers within the range $(-\beta, \beta)$ to $\{\mathrm{W}(\nu(l), \nu(l+1))\}$ and $\{\mathrm{T}(\nu(l))\}$. To be clear and self-contained, we present the basic computing procedure of a typical ANN constructed by the classic fully-connected architecture and relying on the back-propagation mechanism based neural computing as follows.

Assuming there are $\eta(1)$ input data samples denoted as $\{\mathrm{X}(\nu(1))\}$, the input layer does not manipulate these data samples but only transfers them to the hidden layers for computing, which results in the output of the neurons in the input layer as $\mathrm{Y}(\nu(1))=\mathrm{X}(\nu(1))$. Subsequently, the neurons in the hidden layers and the output layer collect the inputs from all neurons in the previous layer and jointly process them to yield the output as

$$
\mathrm{Y}(\nu(l+1))=\mathrm{F}\left(\sum_{\nu(l)=1}^{\eta(l)} \mathrm{W}(\nu(l), \nu(l+1)) \mathrm{Y}(\nu(l))-\mathrm{T}(\nu(l+1))\right),
$$

where $F(\cdot)$ is the activation function for performing neural computing, which can be set as a logistic/sigmoid, sign, step, or constrained linear function, depending on the nature of inputs and computing demands.

The outputs of the neurons located in the output layer are the final outputs of the ANN and will be compared with the desired outputs $\{Z(\nu(L))\}$ to determine the computing error for each neuron in the output layer: $\mathrm{E}(\nu(L))=\mathrm{Z}(\nu(L))-\mathrm{Y}(\nu(L))$. According to the back-propagation mechanism, these computing errors can be further used to recursively calculate the gradient descents and for neurons in the output and hidden layers by $\mathrm{D}(\nu(L))=\mathrm{Y}(\nu(L))(1-\mathrm{Y}(\nu(L))) \mathrm{E}(\nu(L))$ and $\mathrm{D}(\nu(l))=$ $\mathrm{Y}(\nu(l))(1-\mathrm{Y}(\nu(l))) \sum_{\nu(l+1)=1}^{\eta(l+1)} \mathrm{D}(\nu(l+1)) \mathrm{W}(\nu(l), \nu(l+1))$, given $l \in\{1,2, \ldots, L-1\}$, respectively. With gradient descents, 
TABLE I: Technical specification of the primary ANN estimating the on/off states of $N$ subcarriers.

\begin{tabular}{cl}
\hline Attribute & Explanation \\
\hline $\begin{array}{c}\text { Functiona- } \\
\text { lity }\end{array}$ & $\begin{array}{l}\text { Estimate the subcarrier on/off states by the input } \\
\text { amplitudes. }\end{array}$ \\
\hline Denotation & $\begin{array}{l}\text { The number of layers is denoted as } L_{p r}, \text { and the } \\
\text { number of neurons in the } l \text { th layer is denoted as } \eta_{p r}(l) .\end{array}$ \\
\hline Input & There are $N$ inputs that are $\left\{\hat{A}_{n} / \sqrt{P_{T}}\right\}, \forall n \in \mathcal{N}$. \\
\hline Processing & $\begin{array}{l}\text { Use the logistic function } \mathrm{F}_{p r}(x)=1 /(1+\exp (-x)) \text { as } \\
\text { the activation function and gradient descent algorithm to } \\
\text { enable back-propagation and dynamical link weight and } \\
\text { activation threshold adjustments with learning rate } \alpha_{p r} .\end{array}$ \\
\hline Output & $\begin{array}{l}\text { There are } N \text { outputs that are the estimated subcarrier } \\
\text { on/off states }\left\{\hat{\phi}_{n}\right\}, \forall n \in \mathcal{N} .\end{array}$ \\
\hline $\begin{array}{c}\text { Initialization } \\
\text { method }\end{array}$ & $\begin{array}{l}\text { Random initialization for link weights and activation } \\
\text { thresholds with range expansion coefficient } \beta_{p r} .\end{array}$ \\
\hline Termination \\
condition
\end{tabular}$\quad$\begin{tabular}{c} 
All labeled training data sets have been exhaustively used. \\
\hline
\end{tabular}

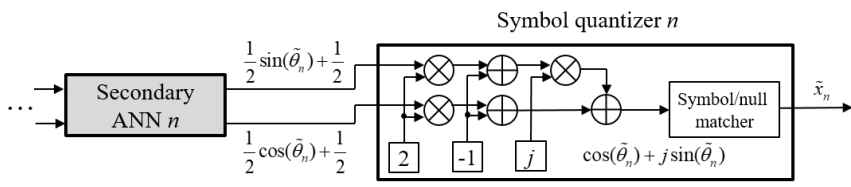

Fig. 3: Processing of the estimated projections of sine and cosine of the phase angle for the original symbol carried on the $n$th subcarrier.

the correction terms for link weights and activation thresholds can be calculated as $\Delta \mathrm{W}(\nu(l), \nu(l+1))=\alpha \mathrm{Y}(\nu(l)) \mathrm{D}(\nu(l+1))$ and $\Delta \mathrm{T}(\nu(l))=-\alpha \mathrm{D}(\nu(l))$, by which back-propagation mechanism iteratively updates the link weights and activation thresholds as $\mathrm{W}(\nu(l), \nu(l+1)) \leftarrow \mathrm{W}(\nu(l), \nu(l+1))+\Delta \mathrm{W}(\nu(l), \nu(l+1))$ and $\mathrm{T}(\nu(l)) \leftarrow \mathrm{T}(\nu(l))+\Delta \mathrm{T}(\nu(l))$ until certain termination conditions are satisfied. Enabled by such an iterative updating procedure, the neurons of the ANN can be trained to produce outputs close to the desired outputs (a.k.a. labels). This is the fundamental working principle of the intelligent functionality for the ANNs considered in this letter.

Although we presented the computing procedure and working principle of a fully-connected ANN above in a general manner, researchers in computer science and signal processing have reached a consensus that there might not exist a general-purpose ANN architecture that can be applied to solve most optimization problems, but each specific optimization problem requires a specially designed ANN architecture [14]. Following this ideology, as signal detection is in nature within the realm of optimization, we propose and expatiate on the architecture designs and functionality for the primary and secondary ANNs in Table I and Table II, respectively.

It should be noted that the outputs of the secondary ANNs are the estimated projections of sine and cosine of a phase angle when a symbol is transmitted on the $n$th subcarrier, instead of the sinusoidal functions per se. This is because the logistic function can only output values within $(0,1)$ that is linearly projected back to $(-1,1)$ by the mapping relation $\mathcal{F}:(0,1) \stackrel{Y=2 X-1}{\longrightarrow}(-1,1)$ in the following symbol quantizer to approximate sinusoidal functions. For clarity, we pictorially illustrate the processing details inside the symbol quantizer in Fig. 3.
TABLE II: Technical specification of the $n$th secondary ANN estimating the original symbol or a null conveyed on the $n$th subcarrier.

\begin{tabular}{cl}
\hline Attribute & Explanation \\
\hline $\begin{array}{c}\text { Functiona- } \\
\text { lity }\end{array}$ & $\begin{array}{l}\text { Estimate the projections of sine and cosine of the phase } \\
\text { angle of the symbol or a null carried on the } n \text {th subcarrier. }\end{array}$ \\
\hline Denotation & $\begin{array}{l}\text { The number of layers is denoted as } L_{s e} \text {, and the } \\
\text { number of neurons in the } l \text { th layer is denoted as } \eta_{s e}(l) .\end{array}$ \\
\hline Input & There are two inputs that are $\hat{\theta}_{n} /(2 \pi)$ and $\tilde{\phi}_{n}$. \\
\hline Processing & $\begin{array}{l}\text { Use the logistic function } \mathrm{F}_{\text {se }}(x)=1 /(1+\exp (-x)) \text { as } \\
\text { the activation function and gradient descent algorithm to } \\
\text { enable back-propagation and dynamical link weight and } \\
\text { activation threshold adjustments with learning rate } \alpha_{s e} .\end{array}$ \\
\hline Output & $\begin{array}{l}\text { There are two outputs that are the estimated projections } \\
\text { of sine and cosine of the phase angle for the original } \\
\text { symbol i.e., } \frac{1}{2} \text { sin }\left(\tilde{\theta}_{n}\right)+\frac{1}{2} \text { and } \frac{1}{2} \cos \left(\tilde{\theta}_{n}\right)+\frac{1}{2} \text { or } \frac{1}{2} \\
\text { and } \frac{1}{2} \text { for a null. }\end{array}$ \\
\hline $\begin{array}{c}\text { Initialization } \\
\text { method }\end{array}$ & $\begin{array}{l}\text { Random initialization for link weights and activation } \\
\text { thresholds with range expansion coefficient } \beta_{s e} .\end{array}$ \\
\hline condition & All labeled training data sets have been exhaustively used. \\
\hline
\end{tabular}

\section{Numerical Simulations and Discussion}

\section{A. Performance Evaluation Metrics}

By the smart detector, the holistic estimation procedure of OFDM-SNM is split into two stages for the SNP and the constellation symbols or nulls. We are interested in both of the quantized intermediate estimates given by the primary ANN and the quantized final estimated block given by the secondary ANNs. To evaluate the performance from both qualitative and quantitative perspectives, we define the the primary error rate (PER) $P_{p r}=\mathbb{P}\left\{\tilde{\mathbf{x}}_{\mathrm{SNP}}(k) \neq \mathbf{x}_{\mathrm{SNP}}(k)\right\}$ and the primary mean squared error (PMSE) $E_{p r}=\mathbb{E}\left\{\left\|\tilde{\mathbf{x}}_{\mathrm{SNP}}(k)-\mathbf{x}_{\mathrm{SNP}}(k)\right\|_{F}^{2}\right\}$, where $\|\cdot\|_{F}$ denotes the Frobenius norm of the enclosed matrix/vector. Likewise for the secondary ANN, we define the secondary error rate (SER) $P_{\text {se }}=\mathbb{P}\{\tilde{\mathbf{x}}(k) \neq \mathbf{x}(k)\}$ and the secondary mean squared error (SMSE) $E_{p r}=\mathbb{E}\left\{\|\tilde{\mathbf{x}}(k)-\mathbf{x}(k)\|_{F}^{2}\right\}$ for qualitative and quantitative evaluation purposes.

\section{B. Simulation Configurations}

For simplicity, we normalize the ANN hyperparameters $\alpha_{p r}=$ $\alpha_{s e}=1$ and $\beta_{p r}=\beta_{s e}=1$. We further assume an AWGN channel with static and normalized channel power gains throughout the entire training phase. Thus, we can randomly generate complex noise terms abiding distribution $\mathcal{C N}\left(0, N_{0}\right)$ and the labeled training data samples by (1). The total number of labeled training data samples is $3 \times 10^{4}$, and the number of repeated trials for averaging the training performance is $10^{4}$. For the ANN architectures, we set $L_{p r}=4$ and $\left[\eta_{p r}(1), \eta_{p r}(2), \eta_{p r}(3), \eta_{p r}(4)\right]^{T}=$ $[N, 10 N, 10 N, N]^{T}$ for the primary ANN and $L_{s e}=4$ and $\left[\eta_{p r}(1), \eta_{s e}(2), \eta_{s e}(3), \eta_{s e}(4)\right]^{T}=[2,20,20,2]^{T}$ for all $N$ secondary ANNs. In simulations, we stipulate $N=4, M=2$ (BPSK), and $P_{T} / N_{0}=10 \mathrm{~dB}$, which represent simple wireless systems with a strict transmit power constraint in the IoT and machine-type communication (MTC) networks.

\section{Discussion of Numerical Results}

We illustrate the qualitative and quantitative results by numerical simulations in Fig. 4 and Fig. 5, respectively. From both figures, 


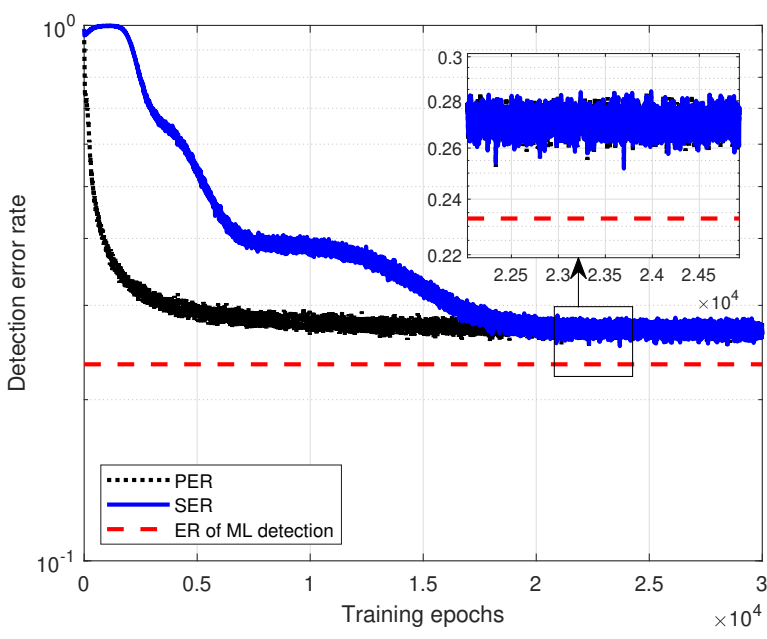

Fig. 4: Detection error rates vs. training epochs.

the all-important observation is that learning through an adequate number of labeled data samples, the proposed smart detector using the CANN can achieve comparable detection performance to the optimal ML detector. This is aligned with our informationtheoretic analysis of the detection for OFDM-SNM in Section III-A. The training saturation happens after being trained for $2 \times 10^{4}$ epochs with different labeled data samples. These results validate the effectiveness and efficiency of the proposed smart detector. In this way, we can divide the holistic OFDMSNM detection procedure into a two-stage detection procedure on a per-subcarrier basis and thereby decrease the computational complexity from the exponential scale $\mathcal{O}\left(M^{N}\right)$ to the polynomial scale $^{4} \mathcal{O}\left(M N^{2}\right)$. For a given channel condition, as the offline ANN training is only required once and for all, the computational advantage of the CANN based detection indicates a much fast online detection procedure, especially when $M$ and $N$ become large. Such a setup can effectively release the requirement of initial subcarrier grouping aiming at reducing $N$ for suppressing the detection complexity for the ML detection. Meanwhile, comparing PER/PMSE and SER/SMSE, we can also find that the estimation of SNP is relatively easy, while the bottleneck is the estimations of symbols or nulls.

\section{CONCLUSION}

In this letter, we employed a novel paradigm called CANN at the OFDM-SNM receiver to realize low-complexity detection. The receiver equipped with the CANN framework and other auxiliary modules can split the integrated detection procedure into two sequential detection procedures for on/off states and symbols. In this way, the computational complexity of the OFDM-SNM detection can be reduced from the exponential scale to the polynomial scale, whereas, as verified by numerical simulations, the detection

\footnotetext{
${ }^{4}$ As illustrated in Fig. 1, once the CANN has been solidified after training, the online processing of received signal vectors is dominated by the primary and secondary ANNs, so as the computational complexity incurred. The number of possible outputs of the primary ANN is $N$, and the for each secondary ANN, the number of possible outputs is $M+1$. As there exist $N$ secondary ANNs, the total computational complexity required for detecting one received signal vector can be expressed as $\mathcal{O}\left(M N^{2}\right)$ by neglecting low-order terms.
}

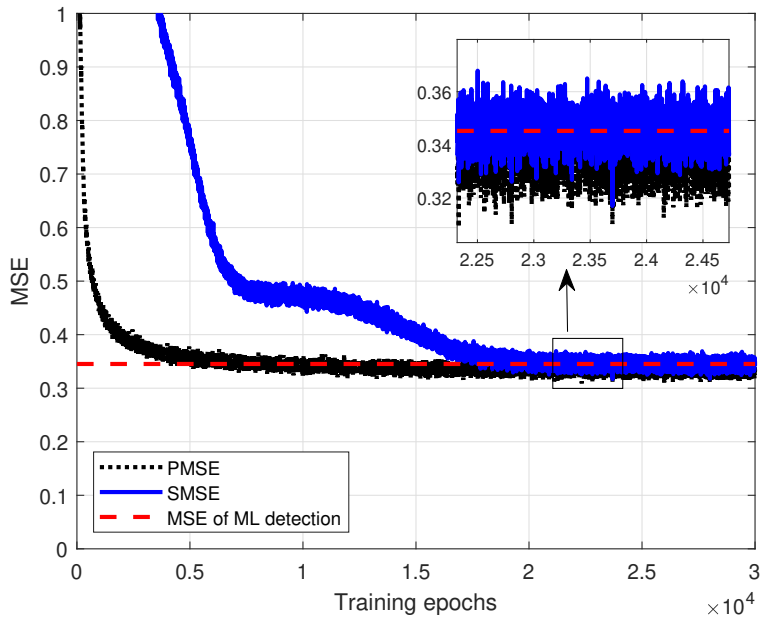

Fig. 5: MSEs vs. training epochs.

performance is still comparable to that provided by the optimal ML detection. As a general paradigm, the design principle and ideas of CANN presented in this letter can also be extended to other complicated modulation schemes if their detection procedures can be split into a limited number of decoupled steps.

\section{REFERENCES}

[1] A. M. Jaradat, J. M. Hamamreh, and H. Arslan, "Modulation options for OFDM-based waveforms: Classification, comparison, and future directions," IEEE Access, vol. 7, pp. 17263-17278, 2019.

[2] E. Basar, M. Wen, R. Mesleh, M. Di Renzo, Y. Xiao, and H. Haas, "Index modulation techniques for next-generation wireless networks," IEEE Access, vol. 5, pp. 16 693-16746, 2017.

[3] J. M. Hamamreh, E. Basar, and H. Arslan, "OFDM-subcarrier index selection for enhancing security and reliability of 5G URLLC services," IEEE Access, vol. 5, pp. 25 863-25875, 2017.

[4] T. Mao, Q. Wang, Z. Wang, and S. Chen, "Novel index modulation techniques: A survey," IEEE Commun. Surv. Tutor, vol. 21, no. 1, pp. 315-348, 2019.

[5] J. Li, S. Dang, M. Wen, X. Jiang, Y. Peng, and H. Hai, "Layered orthogonal frequency division multiplexing with index modulation," IEEE Systems Journal, vol. 13, no. 4, pp. 3793-3802, 2019.

[6] A. M. Jaradat, J. M. Hamamreh, and H. Arslan, "OFDM with subcarrier number modulation," IEEE Wireless Commun. Lett., vol. 7, no. 6, pp. 914 917, 2018.

[7] S. Dang, G. Ma, B. Shihada, and M.-S. Alouini, "Enhanced orthogonal frequency-division multiplexing with subcarrier number modulation," IEEE Internet of Things Journal, vol. 6, no. 5, pp. 7907-7920, 2019.

[8] S. Dang, J. Zhou, B. Shihada, and M. S. Alouini, "Relay assisted OFDM with subcarrier number modulation in multi-hop cooperative networks," IEEE Wireless Commun. Lett., vol. 9, no. 11, pp. 1869-1873, 2020.

[9] A. A. Zaidi, R. Baldemair, V. Moles-Cases, N. He, K. Werner, and A. Cedergren, "OFDM numerology design for 5G new radio to support IoT, eMBB, and MBSFN," IEEE Commun. Standards Mag., vol. 2, no. 2, pp. 78-83, 2018.

[10] A. M. Jaradat, J. M. Hamamreh, and H. Arslan, "OFDM with hybrid number and index modulation," IEEE Access, vol. 8, pp. 55 042-55 053, 2020.

[11] S. Dang, M. Wen, S. Mumtaz, J. Li, and C. Li, "Enabling multi-carrier relay selection by sensing fusion and cascaded ANN for intelligent vehicular communications," IEEE Sensors Journal, 2020.

[12] Y. Yang and B. Jiao, "Information-guided channel-hopping for high data rate wireless communication," IEEE Commun. Lett., vol. 12, no. 4, pp. 225-227, 2008.

[13] M. Negnevitsky, Artificial Intelligence: A Guide to Intelligent Systems. Addison-Wesley, 2005.

[14] M. Chen, U. Challita, W. Saad, C. Yin, and M. Debbah, "Artificial neural networks-based machine learning for wireless networks: A tutorial," IEEE Commun. Surv. Tutor, vol. 21, no. 4, pp. 3039-3071, 2019. 